Pacific Journal of Mathematics

ASYMPTOTICS. III. STATIONARY PHASE FOR TWO
PARAMETERS WITH AN APPLICATION TO BESSEL

DUANE BATHE 


\section{ASYMPTOTICS III: STATIONARY PHASE FOR TWO PARAMETERS WITH AN APPLICATION TO BESSEL FUNCTIONS}

\section{SATHER}

1. Introduction. The method of stationary phase has long been a valuable analytical tool for investigating the asymptotic behavior as $p \rightarrow \infty$ of integrals of the form

$$
I(p)=\int_{0}^{a} Q(t) \exp (i p F(t)) d t .
$$

As a natural generalization of the method of stationary phase involving one parameter we will investigate the asymptotic behavior of an integral of the form

$$
I(h, k)=\int_{0}^{a} t^{\gamma-1} q(t) \exp \left[i\left(h t^{\lambda} f(t)+k t^{\nu} g(t)\right)\right] d t
$$

where $h$ and $k$ tend to infinity independently.

It will be shown that under certain restrictions between the real numbers $\lambda, \nu$ and $\gamma$ that the asymptotic form of $I(h, k)$ is determined by the behavior of the ratio $k h^{-\nu / \lambda}$ as $h, k \rightarrow \infty$ and by the character of $f$ and $g$ in a neighborhood of $t=0$. For example, if $\gamma<\nu<\lambda$, $\gamma>0, f(0)>0, g(0)>0$ and $k h^{-\nu / \lambda} \rightarrow \infty$ then

$$
I(h, k) \backsim \frac{q(0) \Gamma\left(\frac{\gamma}{\nu}\right) \exp \left(\frac{i \pi \gamma}{2 \nu}\right)}{\nu[k g(0)]^{\gamma / \nu}} .
$$

As an immediate application of our results we will determine the asymptotic behavior of the Bessel function $J_{\nu}(x)$ in Watson's transition region, i.e. when $\nu, x$ and $|\nu-x|$ are large and $\nu / x$ is nearly equal to 1. In particular, we will obtain a simple rigorous proof of Nicholson's formulas under the restriction that $0<\lim \sup x^{-1 / 3}|\nu-x|<\infty$.

2. General assumptions. Throughout the paper we shall use $A \backsim B$ to mean $\lim A / B=1$, and all limits will mean the limit as $h$ and $k$ tend to infinity. A similar remark applies to order symbols.

We shall consider $I(h, k)$ under the following general assumptions:

Received January 3,1962. This paper was written at the University of Minnesota in part under Contract Nonr 710(16), sponsored by the Office of Naval Research, and in part under a National Science Foundation Fellowship. The author wishes to express his appreciation to Professor W. Fulks for suggesting the problem and for giving valuable aid in its solution. 
(i ) $k=o(h)$,

(ii) $\lambda>0, \nu>0$, and $\gamma>0$,

(iii) $q(0) \neq 0$ and $g(0) \neq 0$,

(iv) $f, g$ and $q$ are real valued functions such that $f \in C^{2}, g \in C^{2}$ and $q \in C$ on $[0, a]$,

(v) $\lambda f(t)+t f^{\prime}(t)>0$ on $[0, a]$.

For convenience we shall consider here only the case $f(0)>0$. If $f(0)<0$ and $-f$ satisfies certain obvious conditions one obtains analogous results with $-i$ and $-g$ replacing $i$ and $g$, respectively.

3. Preliminary lemmas. We shall first establish the following lemmas.

Lemma I. Consider $I(p)=\int_{0}^{b} \omega(t) \Psi(t) \exp (i p \Phi(t)) d t$. Suppose $d$ is a nonnegative constant and $p, \alpha$ and $\mu$ are functions of $h$ and $k$ such that $p \rightarrow \infty, \mu \rightarrow 0$ and $\alpha$ is bounded as $h, k \rightarrow \infty$.

(i) $\Phi(t)=t^{r} \phi(\alpha t), \Psi(t)=t^{s-1} \psi(\mu(t+d))$, the functions $\phi$ and $\psi$ are real with $\psi(0) \neq 0, \quad r>0,0<s<r, \phi(\alpha t)>0$ for $0 \leqq t \leqq c^{\prime}$, $c^{\prime}>0$ and $\phi \in C^{n+2}$ and $\psi \in C^{n}$ for $0 \leqq t \leqq c^{\prime}$ where $m$ and $n$ are the least integers such that $m r>1$ and $n \geqq m(r-s)+1$, respectively.

(ii) $b$ is a constant such that $0<b<c^{\prime}$ and $b M K / m_{0} r<1$ where $M=\operatorname{maximum}_{0 \leqq t \leqq c t}\left|\phi^{\prime}(t)\right|, m_{0}=\operatorname{minimum}_{0 \leqq t \leqq c} \phi(t)$ and $K \geqq \alpha$ when $h, k$ are sufficiently large.

(iii) $\omega=u+i v$ is a complex valued function such that $u(0)=1$, $v(0)=0$ and $u, v \in C^{n}$ for $0 \leqq t \leqq c^{\prime}$. Then

$$
I(p) \backsim \frac{\psi(0) \Gamma\left(\frac{s}{r}\right) \exp \left(\frac{i \pi s}{2 r}\right)}{r[p \phi(0)]^{s / r}} .
$$

Proof. We may set $v \equiv 0$ since it will be seen that the contribution from $v$ to $I(p)$ is negligible because $v(0)=0$. Let $x=t[\phi(\alpha t)]^{1 / r}$. Since $x^{\prime}(t)>0$ for $0 \leqq t \leqq b$ and $x \in C^{n+2}$ there exists a unique inverse function, say $t(x)$, such that $t \in C^{n+2}$ for $0 \leqq x \leqq b[\phi(\alpha b)]^{1 / r}=a, t(0)=0$ and $t^{\prime}(0)=a_{1}=[\phi(0)]^{-1 / r}$. Hence we may write $t(x)=a_{1} x+a_{2} x^{2}+\cdots+$ $a_{n-1} x^{n-1}+A(x) x^{n}$ where $A \in C^{2}$ and $a_{l}$ is bounded as $h, k \rightarrow \infty$ for $2 \leqq l \leqq n-1$. We may assume that $c^{\prime}$ is sufficiently small such that if $t(x)=a_{1} x(1+w(x))$ then $|w(x)|<1$ for $0 \leqq x \leqq a$. This implies that

$$
(t(x))^{s-1}=a_{1}^{s-1}\left(1+b_{1} x+\cdots+b_{n-2} x^{n-2}+z(x) x^{n-1}\right) x^{s-1}
$$

where $z \in C$ and $b_{l}$ is independent of $x$ for $1 \leqq l \leqq n-2$. If we now expand $\psi$ and $\omega$ about $t=0$ and substitute $t(x)$, and let $B(x)=\omega(t(x))$ $(t(x))^{s-1} \psi(\mu(t(x)+d))$ we have

$$
B(x) \frac{d t(x)}{d x}=a_{1}^{s} u(\mu d) x^{s-1}+c_{0} x^{s}+\cdots+c_{n-3} x^{s+n-3}+D(x) x^{n+s-2}
$$


where $h$ and $k$ are sufficiently large such that $\mu d<b, D \in C$ and $c_{l}$ is bounded as $h, k \rightarrow \infty$ and independent of $x$ for $0 \leqq l \leqq n-3$. Therefore,

$$
I(p)=a_{1}^{s} \psi(\mu d) \int_{0}^{a} x^{s-1} \exp \left(i p x^{r}\right) d x+J(p)+\sum_{l=0}^{n-3} c_{l} \int_{0}^{a} x^{s+l} \exp \left(i p x^{r}\right) d x
$$

where $J(p)=\int_{0}^{a} D(x) x^{n+s-2} \exp \left(i p x^{r}\right) d x . \quad$ Since $\int_{0}^{\infty} e^{i t} t^{\beta-1} d t=\exp (i \pi \beta / 2) \Gamma(\beta)$ for $0<\beta<1$ and $r \leqq n+s-1<r+1$ when $r>1$ we have

$$
\begin{aligned}
I(p) & =\frac{a_{1}^{s} \psi(\mu d) \exp \left(\frac{i \pi s}{2 r}\right) \Gamma\left(\frac{s}{r}\right)}{r p^{s / r}}+J(p)+o\left(p^{-s / r}\right) \\
& =\frac{a_{1}^{s} \psi(0) \exp \left(\frac{i \pi s}{2 r}\right) \Gamma\left(\frac{s}{r}\right)}{r p^{s / r}}+J(p)+o\left(p^{-s / r}\right) .
\end{aligned}
$$

Finally an integration by parts yields $J(p)=0(1 / p)$ since $n-(r+1-s) \geqq 0$ by the choice of $n$ and $D \in C$. This completes the proof of Lemma I for the case $r>1$. For $0<r \leqq 1$ one makes the change of variable $t=x^{m}$ and the desired result follows from the case $r>1$.

Lemma II. Suppose that in addition to the assumptions of Lemma $I$ that $r$ is an even integer, $s=1, \phi(\alpha t)>0$ for $-c^{\prime} \leqq t \leqq c^{\prime}, b$ satisfies the same conditions as in Lemma I except that $M$ and $m_{0}$ are now determined for $-c^{\prime} \leqq t \leqq c^{\prime}$, and $\omega$, $\psi$ and $\phi$ are now in their respective differentiability classes given in Lemma I for $-c^{\prime} \leqq t \leqq c^{\prime}$. Then

$$
\int_{-b}^{b} \omega(t) \Psi(t) \exp (i p \Phi(t)) d t \backsim \frac{2 \psi(0) \Gamma\left(\frac{1}{r}\right) \exp \left(\frac{i \pi}{2 r}\right)}{r[p \phi(0)]^{1 / r}} .
$$

The proof follows immediately from Lemma I.

We will introduce the following functions which will be used throughout the remainder of the paper:

$$
F(t)=t^{\lambda} f(t), G(t)=t^{\nu} g(t) \text { and } Q(t)=t^{\gamma-1} q(t) .
$$

LEMMA III. Under the general assumptions on $F, G$ and $Q$ we have for each arbitrarily small but fixed positive constant $c<a$ that

$$
L(h, k)=\int_{c}^{a} Q(t) \exp [i(h F(t)+k G(t))] d t=0(1 / h) .
$$

Proof. Let $H(t)=F(t)+(k / h) G(t)$. Then $H^{\prime}(t)>0$ for $c \leqq t \leqq a$ and $h, k$ sufficiently large since $\lambda f(t)+t f^{\prime}(t)>0$ by hypothesis and 
$k=o(h)$. Hence an integration by parts implies $L(h, k)=0(1 / h)$.

This completes the necessary lemmas and the main results of the paper will now be presented.

4. The asymptotic evaluation of $I(h, k)$. We shall first consider the case where $k h^{-\nu / \lambda} \rightarrow 0$ so that $I(h, k)$ is almost completely determined by the character of $h f$ at the origin.

\section{THEOREM I. Suppose that}

1. $f \in C^{n+2}$ and $q \in C^{n}$ for $0 \leqq t \leqq c, c>0$, where $m$ and $n$ are the least integers such that $m \lambda>1$ and $n \geqq m(\lambda-\gamma)+1$, respectively,

2. if $0 \leqq t \leqq c$ then $t^{\nu} g(\beta t)=b_{0}+b_{1} t+\cdots+b_{n-2} t^{n-2}+B(t) t^{n-1}$ where $B \in C$ and $b_{l}$ is bounded as $\beta \rightarrow 0$ for $0 \leqq l \leqq n-2$,

3. $k^{\lambda}=o\left(h^{\nu}\right)$ and $\gamma<\lambda$. Then

$$
I(h, k) \backsim \frac{q(0) \Gamma\left(\frac{\gamma}{\lambda}\right) \exp \left(\frac{i \pi \gamma}{2 \lambda}\right)}{\lambda[h f(0)]^{\gamma / \lambda}} .
$$

Proof of Theorem I. For $c$ as given we have

$$
I(h, k)=\int_{0}^{c}+\int_{c}^{a}=I^{\prime}(h, k)+0(1 / h)
$$

by Lemma III. Let $t=x k^{-1 / \nu}, \widetilde{f}(x)=f\left(x k^{-1 / \nu}\right), \quad \widetilde{g}(x)=g\left(x k^{-1 / \nu}\right), \widetilde{Q}(x)=$ $Q\left(x k^{-1 / \nu}\right)$ and $p=h k^{-\lambda / \nu}$. For any $b$ such that $0<b<c$ we have

$$
\begin{aligned}
I^{\prime}(h, k) & =k^{-1 / \nu} \int_{0}^{b}\left[\widetilde{Q}(x) \exp \left(i \widetilde{g}(x) x^{\nu}\right)\right] \exp \left(i p \widetilde{f}(x) x^{\lambda}\right) d x \\
& +k^{-1 / \nu} \int_{b}^{c k^{1 / \nu}}=I^{\prime \prime}(h, k)+J(h, k), \text { respectively. }
\end{aligned}
$$

Set $\mu=k^{-1 / \nu}, \psi=q, \phi=f, \lambda=r, \gamma=s, \omega(x)=\exp \left(i x^{\nu} \widetilde{g}(x)\right)$ and note that $f(0)>0$ implies that $\widetilde{f}(x)>0$ for $0 \leqq x \leqq c^{\prime}, c^{\prime}>0$, so that $b$ may be chosen to satisfy the requirements of Lemma I. Hence by Lemma I

$$
I^{\prime \prime}(h, k) \backsim \frac{q(0) \Gamma(\gamma / \lambda) \exp (i \pi \gamma / 2 \lambda)}{\lambda[h f(0)]^{\gamma / \lambda}} .
$$

Therefore to complete the proof of Theorem I it is sufficient to show that $h^{\gamma / \lambda} J(h, k)=o(1)$. Let $d=b k^{-1 / \nu}, H(t)=F(t)=+(k / h) G(t)$ and $P(t)=\lambda f(t)+t f^{\prime}(t)+k t^{\nu-\lambda} / h\left[\nu g(t)+g^{\prime}(t) t\right]$. Note that $P(d) \rightarrow \lambda f(0)=$ $2 B>0$ as $h, k \rightarrow \infty$ since $k^{\lambda}=0\left(h^{\nu}\right)$ and $P(t)$ is continuous for $0<d \leqq t \leqq a$. We may assume that $c$ is such that for $h, k$ sufficiently large, $P(t) \geqq B$ for the entire closed interval $d \leqq t \leqq c$. This implies $H^{\prime}(t) \geqq B t^{\lambda-1}>0$ for $0<d \leqq t \leqq c$ and hence we can integrate $J(h, k)$ by parts as follows; 


$$
\begin{aligned}
J(h, k)= & \int_{d}^{c} Q(t) \exp (i h H(t)) d t=\frac{Q(c) \exp (i h H(c))}{i h H^{\prime}(c)}-\frac{Q(d) \exp (i h H(d))}{i h H^{\prime}(d)} \\
& -\frac{1}{i h} \int_{d}^{c} \frac{Q^{\prime}(t) \exp (i h H(t)) d t}{H^{\prime}(t)}+\frac{1}{i h} \int_{d}^{c} \frac{Q(t) H^{\prime \prime}(t) \exp (i h H(t)) d t}{\left(H^{\prime}(t)\right)^{2}} \\
= & 0(1 / h)+A+J^{\prime}(h, k), \text { respectively. }
\end{aligned}
$$

Using the estimates $H^{\prime}(t) \geqq B t^{\lambda-1}$ and $\left|H^{\prime \prime}(t)\right| \leqq K t^{\lambda-2}$ for some $K$ we see immediately that $J=0\left(k^{(\lambda-\gamma) \nu} / h\right)$. Since $k^{\lambda}=o\left(h^{\nu}\right)$ this implies $h^{\gamma / \lambda} J(h, k)=o(1)$ which completes the proof of Theorem I.

We state the following corollary to Theorem I which may apply when $t^{\nu} g(\beta t)$ does not have the required smoothness at the origin but $f, g$ and $q$ are highly differentiable on $[0, c], c>0$.

Corollary. Suppose that $\nu+\gamma>\lambda$ and

1. $f \in C^{n+2}, g \in C^{n}$ and $q \in C^{n}$ for $0 \leqq t \leqq c, c>0$ where $m$ and $n$ are the least integers such that $m(\nu+\gamma-\lambda) \geqq 2, m \lambda>1$ and $n \geqq m(\lambda-\gamma)+1$, 2. $k^{\lambda}=o\left(h^{\nu}\right)$ and $\gamma<\lambda$. Then

$$
I(h, k) \backsim \frac{q(0) \Gamma\left(\frac{\gamma}{\lambda}\right) \exp \left(\frac{i \pi \gamma}{2 \lambda}\right)}{\lambda[h f(0)]^{\gamma / \lambda}} .
$$

Proof. Note that $m \nu \geqq m(\lambda-\gamma)+2>n$ by the definition of $n$ and hence $x^{m \nu} \in C^{n}$. The change of variable $t=x^{m}$ and the use of Theorem I completes the proof.

We shall next consider the case where the behavior of $k g$ at the origin becomes a significant factor in the asymptotic evaluation of $I(h, k)$.

\section{THEorem II. Suppose that}

1. $q \in C^{n}$ and $g \in C^{n+2}$ for $0 \leqq t \leqq c, c>0$, where $m$ and $n$ are the least integers such that $m \nu>1$ and $n \geqq m(\nu-\gamma)+1$, respectively, 2. if $0 \leqq t \leqq c$ then $t^{\lambda} f(\beta t)=b_{0}+b_{1} t+\cdots+b_{n-2} t^{n-2}+B(t) t^{n-1}$ where $B \in C$ and $b_{l}$ is bounded as $\beta \rightarrow 0$ for $0 \leqq l \leqq n-2$,

3. $g(0)>0, h^{\nu}=o\left(k^{\lambda}\right)$ and $\gamma<\nu<\lambda$. Then

$$
I(h, k) \backsim \frac{q(0) \Gamma\left(\frac{\gamma}{\nu}\right) \exp \left(\frac{i \pi \gamma}{2 \nu}\right)}{\nu[k g(0)]^{\gamma / \nu}} .
$$

Proof of Theorem II. The proof of Theorem II follows from the proof of Theorem I with the roles of $f$ and $g, \lambda$ and $\nu, h$ and $k$ intercharged. 
CoRollary. Suppose that

1. $f \in C^{n}, q \in C^{n}$ and $g \in C^{n+2}$ for $0 \leqq t \leqq c, c>0$, where $m$ and $n$ are the least integers such that $m(\lambda+\gamma-\nu) \geqq 2, m \nu>1$ and $n \geqq m(\nu-\gamma)+1$, 2. $g(0)>0, h^{\nu}=o\left(k^{\lambda}\right)$ and $\gamma<\nu<\lambda$. Then

$$
I(h, k) \backsim \frac{q(0) \Gamma\left(\frac{\gamma}{\nu}\right) \exp \left(\frac{i \pi \gamma}{2 \nu}\right)}{\nu[k g(0)]^{\gamma / \nu}} .
$$

When $k h^{-\nu / \lambda} \rightarrow \infty$ and $g(0)<0$ the character of both $F$ and $G$ in a neighborhood of $t=0$ becomes important since for $h$ and $k$ sufficiently large they determine uniquely in some $\left(0, c_{0}\right)$ a number $\tau$ such that $h F^{\prime}(\tau)+k G^{\prime}(\tau)=0$ and in terms of which the asymptotic form of $I(h, k)$ may be expressed.

THEOREM III. Suppose that $g(0)<0, \nu<\lambda, \gamma<\lambda, h^{\nu}=o\left(k^{\lambda}\right), f \in C^{6}$, $g \in C^{6}$ and $q \in C^{2}$ for $0 \leqq t \leqq c, c>0$, and hypothesis 1 and 2 Theorem II are satisfied when $\nu \geqq \gamma$.

A. If $\nu<2 \gamma$ then

$$
\begin{aligned}
& I(h, k) \backsim \frac{\sqrt{2} q(0) \Gamma\left(\frac{1}{2}\right) \exp \left(\frac{i \pi}{4}\right)}{(\lambda-\nu)^{1 / 2}}\left[\frac{(\lambda h f(0))^{\nu-2 \gamma}}{(-\nu k g(0))^{\lambda-2 \gamma}}\right]^{1 / 2(\lambda-\nu)} \\
& \quad \times \exp [i(h F(\tau)+k G(\tau))] .
\end{aligned}
$$

B. If $\nu=2 \gamma$ then

$$
I(h, k) \backsim \frac{q(0) \Gamma\left(\frac{1}{2}\right) \exp \left(\frac{i \pi}{4}\right)}{(-\nu k g(0))^{1 / 2}}\left\{\frac{\sqrt{2} \exp [i(h F(\tau)+k G(\tau))]}{(\lambda-\nu)^{1 / 2}}-i \nu^{-1 / 2}\right\} .
$$

C. If $\nu>2 \gamma$ then

$$
I(h, k) \backsim \frac{q(0) \Gamma\left(\frac{\gamma}{\nu}\right) \exp \left(\frac{\gamma \pi}{2 \nu i}\right)}{\nu[-k g(0)]^{\gamma / \nu}} .
$$

Proof of Theorem III. We may assume that $c$ is such that $G^{\prime}(t)<0$ and $f(t)>0$ for $0<t \leqq c$. For $0<t \leqq c$ let $D(t)=F^{\prime}(t) /-G^{\prime}(t)$ with $D(0)=0$. Then $D^{\prime}(t)=t^{\lambda+\nu-\xi} /\left(G^{\prime}(t)\right)^{2}[\nu \lambda f(t) g(t)(\nu-\lambda)+t E(t)]$ for $0<t \leqq c$ where $E$ is continuous on $[0, c]$. Hence there exists $c_{0}$ such that $0<c_{0}<c, D^{\prime}(t)>0$ for $0<t \leqq c_{0}, D\left(c_{0}\right)>0$ and for $h$ and $k$ sufficiently large $k / h<D\left(c_{0}\right)$. This implies that there exists a unique $\tau \in\left(0, c_{0}\right)$ such that $D(\tau)=k / h$ which is equivalent to $h F^{\prime}(\tau)+k G^{\prime}(\tau)=0$. Moreover from the definition of $D$ we have

$$
\tau=\left(\frac{-\nu k g(0)}{\lambda h f(0)}\right)^{1 / \lambda-\nu}(1+o(1))
$$


which implies that $\tau^{\lambda-\nu}=o(k / h)=o(1)$.

If we now let $H(t)=F(t)+(k / h) G(t)$ and expand $h(H(t)-H(\tau))$ about $t=\tau$ we have using the integral form of the remainder

$$
\begin{aligned}
h(H(t)-H(\tau)) & =h \int_{\tau}^{t}(t-y) F^{\prime \prime}(y) d y+k \int_{\tau}^{t}(t-y) G^{\prime \prime}(y) d y \\
& =h R(t, \tau)+k S(t, \tau), \text { respectively. }
\end{aligned}
$$

We may further assume that $c_{0}$ is so small that $f, f^{\prime}, f^{\prime \prime}, g, g^{\prime}$ and $g^{\prime \prime}$ are of constant sign for $0 \leqq t \leqq c_{0}$. If we apply the mean value theorem for integrals and substitute $t=\tau(x+1)$ we have for $-1<x<1$ that

$$
\begin{aligned}
& T(x, \tau)=R(\tau(x+1), \tau)=\frac{\tau^{\lambda} x^{2}}{2}\left[\lambda(\lambda-1) f\left(\tau_{0}(x)\right) \alpha_{0}(x)\right. \\
& \left.\quad+\tau(\lambda+1) \alpha_{1}(x) f^{\prime}\left(\tau_{1}(x)\right)+\tau^{2} f^{\prime \prime}\left(\tau_{2}(x)\right) \alpha_{2}(x)\right]=\frac{\tau^{\lambda} x^{2}}{2} P_{1}(x)
\end{aligned}
$$

where $\alpha_{0}, \alpha_{1}, \alpha_{2} \in C^{\infty}, \alpha_{0}(0)=\alpha_{2}(0)=\alpha_{1}(0)=1, P_{1} \in C^{4}$ and $P_{1}(0)=\lambda(\lambda-1)$ $f(0)+o(1)$. Similarly

$$
W(x, \tau)=S(\tau(x+1), \tau)=\frac{1}{2} \tau^{\nu} x^{2} P_{2}(x)
$$

where $P_{2} \in C^{4}$ and $P_{2}(0)=\nu(\nu-1) g(0)+o(1)$. Let $d_{0}=\left(c_{0} / \tau\right)-1, I^{\prime}(h, k)=$ $\exp (-i h H(\tau)) I(h, k)$ and choose $b$ such that $\tau(b+1)<c_{0}$ and $0<b<1$.

$$
I^{\prime}(h, k)=\int_{0}^{c_{0}}+0\left(\frac{1}{h}\right)=I^{\prime \prime}(h, k)+0\left(\frac{1}{h}\right) .
$$

$$
\begin{aligned}
I^{\prime \prime}(h, k) & =\tau \int_{-1}^{-b}+\tau \int_{-b}^{b}+\tau \int_{b}^{a_{0}} Q(\tau(x+1)) \exp [i(h T(x, \tau)+k W(x, \tau))] d x \\
& =L(h, k)+I^{\prime \prime \prime}(h, k)+J(h, k), \text { respectively. }
\end{aligned}
$$

Let

$\mu=\tau, p=(1 / 2) h \tau^{\lambda}, \psi=q, \omega(x)=(1+x)^{\gamma-1}$ and $\phi(x)=P_{1}(x)+\left(k \tau^{\nu-\lambda} / h\right) P_{2}(x)$.

Then $\phi(0)=\lambda(\lambda-\nu) f(0)+o(1)$ implies for $h, k$ sufficiently large that $\phi(x)>0$ for $-c^{\prime} \leqq x \leqq c^{\prime}, c^{\prime}>0$. Hence $b$ may be chosen small enough that the conditions on $b$ in Lemma II are satisfied. Therefore

$$
I^{\prime \prime \prime}(h, k) \backsim \frac{\sqrt{2} q(0) \Gamma\left(\frac{1}{2}\right) \exp \left(\frac{i \pi}{4}\right)}{(\lambda-\nu)^{1 / 2}}\left[\frac{(\lambda h f(0))^{\nu-2 \gamma}}{(-\nu k g(0))^{\lambda-2 \gamma}}\right]^{1 / 2(\lambda-\nu)} .
$$

The contribution of $L(h, k)$ to $I(h, k)$ may be determined by considering

$$
L^{\prime}(h, k)=\int_{0}^{\tau(1-b)} Q(t) \exp (i h H(t)) d t
$$


We note that the uniqueness of $\tau$ in $\left[\varepsilon, c_{0}\right], \varepsilon>0$, implies that $H^{\prime}(t) \neq 0$ on $\varepsilon \leqq t \leqq \tau(1-b)$ for every $\varepsilon>0$. In fact, there exists a number $K>0$ which is independent of $\varepsilon$ and for which we have $\left|H^{\prime}(t)\right| \geqq K(k / h) t^{\nu-1}$ for $\varepsilon \leqq t \leqq \tau(1-b)$.

(i) For $\nu<\gamma$ the usual integration by parts together with the above inequality for $H^{\prime}(t)$ yields that $L^{\prime}(h, k)=o(1 / k)$. Hence $L^{\prime}(h, k)=$ $o\left(h^{\nu-2 \gamma} / k^{\lambda-2 \gamma}\right)^{1 / 2(\lambda-\nu)}$ since $h^{\nu}=o\left(k^{\lambda}\right)$.

(ii) For $\nu>\gamma$ we rewrite $L^{\prime}(h, k)$ as

$$
L^{\prime}(h, k)=\int_{0}^{\tau(1-b)} Q(t) \exp \left\{-i\left[k t^{\nu}(-g(t))+h t^{\lambda}(-f(t))\right]\right\} d t
$$

and apply Theorem II with $-g$ playing the role of $f$. Hence

$$
L^{\prime}(h, k) \backsim \frac{q(0) \Gamma\left(\frac{\gamma}{\nu}\right) \exp \left(\frac{-i \pi \gamma}{2 \nu}\right)}{\nu[-k g(0)]^{\gamma / \nu}} .
$$

(iii) Finally for $\nu=\gamma$ a closer examination of the proof of Lemma I together with the change of variable $t=x h^{-1 / \lambda}$ implies for $p^{\prime}=k / h^{v / \lambda}$ that $L^{\prime}(h, k)=h^{-\gamma / \lambda} 0\left(1 / p^{\prime}\right)=0(1 / k)$.

The given relation $h^{\nu}=o\left(k^{\lambda}\right)$ and the calculation

$$
k^{\gamma / \nu} 0\left(\left(\frac{h^{\nu-2 \gamma}}{k^{\lambda-2 \gamma}}\right)^{1 / 2(\lambda-\nu)}\right)=0\left(\left(\frac{h}{k^{\lambda / \nu}}\right)^{\nu-2 \gamma / 2(\lambda-\nu)}\right)
$$

then imply that $I^{\prime \prime \prime}(h, k)=o\left(k^{-\gamma / \nu}\right)$ if $\nu>2 \gamma$ and $L^{\prime}(h, k)=o\left(\left(h^{\nu-2 \gamma} / k^{\lambda-2 \gamma}\right)^{1 / 2(\lambda-\nu)}\right)$ if $\gamma \leqq \nu<2 \gamma$. When $\nu=2 \gamma$ we note that both $L^{\prime}(h, k)$ and $I^{\prime \prime \prime}(h, k)$ are of the same order so that both terms contribute to $I(h, k)$.

To complete the proof of Theorem III we need only show that $J(h, k)$ is negligible compared to $I^{\prime \prime \prime}(h, k)$. For $P(t)$ defined as in the proof of Theorem I and $d=\tau(b+1)$ we have

$$
P(d)=\lambda f(0)\left[1-(b+1)^{\nu-\lambda}\right](1+o(1)) .
$$

Then $P(d)>0$ for $h$ and $k$ sufficiently large and hence proceeding exactly as in the proof of Theorem I we obtain $H^{\prime}(t) \geqq B t^{\lambda-1}>0$ for $0<d \leqq t \leqq c_{0}$ and $2 B=\lambda f(0)\left[1-(1+b)^{\nu-\lambda}\right]$. We now write

$$
J(h, k)=\int_{d}^{c} Q(t) \exp (i h H(t)) d t
$$

and integrate by parts as in Theorem I to obtain $J(h, k)=0\left(\left(h^{\nu-\gamma} / k^{\lambda-\gamma}\right)^{1 / \lambda-\nu}\right)$. Hence $h^{\nu}=o\left(k^{\lambda}\right)$ implies that

$$
\left(\frac{k^{\lambda-2 \gamma}}{h^{\nu-2 \gamma}}\right)^{1 / 2(\lambda-\nu)} J(h, k)=0\left(\left(\frac{h^{\nu}}{k^{\lambda}}\right)^{1 / 2(\lambda-\nu)}\right)=o(1) .
$$


To obtain the value of $\exp [i(h F(\tau)+k G(\tau))]$ in a more explicit form we need to know more about the exact relation between $h$ and $k$. For example we shall state the following corollary under more stringent assumptions.

COROLlary. If in addition to the above assumptions in Theorem III we have $k^{\lambda+1}=o\left(h^{\nu+1}\right)$ then

$$
\exp [i(h F(\tau)+k G(\tau))] \backsim \exp \left\{\frac{i(\nu-\lambda)}{\lambda \nu}\left[\frac{(-\nu k g(0))^{\lambda}}{(\lambda h f(0))^{\nu}}\right]^{1 / \lambda-\nu}\right\} .
$$

Proof of the Corollary to Theorem III. We will use the same notation as in the proof of Theorem III. If we expand $h H(\tau)$ about the origin and substitute for $\tau$ we have

$$
\begin{aligned}
\exp ((i h H(\tau))= & \exp \left\{i \left[h f(0)\left(\frac{-\nu k g(0)}{\lambda h f(0)}\right)^{\lambda / \lambda-\nu}\right.\right. \\
& \left.\left.+k g(0)\left(\frac{-\nu k g(0)}{\lambda h f(0)}\right)^{\nu / \lambda-\nu}\right]\left[1+0\left(\left(\frac{k}{h}\right)^{1 / \lambda-\nu}\right)\right]\right\} \\
= & \exp \left\{\frac{i(\nu-\lambda)}{\lambda \nu}\left[\frac{(-\nu k g(0))^{\lambda}}{(\lambda h f(0))^{\nu}}\right]^{1 / \lambda-\nu}+0\left(\left(\frac{k^{\lambda+1}}{h^{\nu+1}}\right)^{1 / \lambda-\nu}\right)\right\} .
\end{aligned}
$$

Hence if $k^{\lambda+1}=o\left(h^{\nu+1}\right)$ the Corollary is established.

Finally, we shall consider the case where lim sup $k h^{-\nu / \lambda}$ is bounded away from both 0 and $\infty$.

Theorem IV. Suppose that $\gamma<\lambda, \nu<\lambda$ and $0<\lim$ sup $p<\infty$ where $p=k h^{-\nu / \lambda}$. Then

$$
\begin{aligned}
I(h, k) & \backsim q(0) h^{-\gamma / \lambda} \int_{0}^{\infty} x^{\gamma-1} \exp \left[i\left(f(0) x^{\lambda}+p g(0) x^{\nu}\right)\right] d x \\
& =q(0)\left(\frac{k}{h}\right)^{\gamma / \lambda-\nu} \int_{0}^{\infty} x^{\gamma-1} \exp \left[i p^{\lambda / \lambda-\nu}\left(f(0) x^{\lambda}+g(0) x^{\nu}\right)\right] d x .
\end{aligned}
$$

Proof of Theorem $I V$. We will consider only values of $c>0$ such that (i) $\nu g(t)+t g^{\prime}(t)$ is of constant sign for $0 \leqq t \leqq c$ and (ii) for each $\varepsilon>0$ we have $|q(t)-q(0)|<\varepsilon,|f(t)-f(0)|<\varepsilon$ and $|g(t)-g(0)|<\varepsilon$ for $0 \leqq t \leqq c$. Set $H(t)=F(t)+(k / h) G(t)$ and $I^{\prime}(h, k)=\int_{0}^{c}$ as usual. Let $m=\operatorname{minimum}_{0 \leqq t \leqq a} \lambda f(t)+t f^{\prime}(t)>0, \omega=\lim \sup p$ and $M=\operatorname{maxi-}$ $\operatorname{mum}_{0 \leqq t \leqq a}\left(1,\left|f^{(l)}\right|,|q|,\left|q^{\prime}\right|,\left|g^{(l)}\right|,\left|\nu g(t)+t g^{\prime}(t)\right|\right)$ for $l=0,1,2$. Consider a number $b>1$ chosen such that $b>N=(4 M \omega / m)^{1 /(\lambda-\nu)}$. If $d=b h^{-1 / \lambda}<c$ then for $0<d \leqq t \leqq c$ we have for $g(0)<0$

$$
H^{\prime}(t) \geqq t^{\lambda-1}\left(m-\frac{k M}{h d^{\lambda-\nu}}\right) \geqq t^{\lambda-1}\left(m-\frac{m k}{4 \omega h^{\nu / \lambda}}\right) \geqq \frac{1}{2} m t^{\lambda-1}
$$


since $2 \omega>k h^{-\nu / \lambda}$ for $h$ and $k$ sufficiently large. Hence $H^{\prime}(t) \geqq(1 / 2) m t^{\lambda-1}>0$ for $0<d \leqq t \leqq c$. Let $t=x h^{-1 / \lambda}, \quad \widetilde{q}(x)=q\left(x h^{-1 / \lambda}\right), \widetilde{f}(x)=f\left(x h^{-1 / \lambda}\right)$ and $\widetilde{g}(x)=g\left(x h^{-1 / \lambda}\right)$. Then

$$
\begin{aligned}
h^{\gamma / \lambda} I^{\prime}(h, k) & =\int_{0}^{c h^{1 / \lambda}} x^{\gamma-1} \widetilde{q}(x) \exp \left[i\left(\widetilde{f}(x) x^{\lambda}+p \widetilde{g}(x) x^{\nu}\right)\right] d x \\
& =\int_{0}^{b}+\int_{b}^{c h^{1 / \lambda}}=I^{\prime \prime}(h, k)+J(h, k), \text { respectively. }
\end{aligned}
$$

We will first estimate $J(h, k)$ in terms of the number $b$. Since $H^{\prime}(t) \geqq(1 / 2) m t^{\lambda-1}>0$ for $0<d \leqq t \leqq c$ we may integrate $J(h, k)$ by parts as follows:

$$
\begin{aligned}
J(h, k)= & h^{\gamma / \lambda}\left\{\frac{Q(c) \exp (i h H(c))}{i h H^{\prime}(c)}-\frac{Q(d) \exp (i h H(d))}{i h H^{\prime}(d)}\right. \\
& -\frac{1}{i h} \int_{d}^{c} \frac{Q^{\prime}(t) \exp (i h H(t)) d t}{H^{\prime}(t)}+\frac{1}{i h} \int_{d}^{c} \frac{Q(t) F^{\prime \prime}(t) \exp (i h H(t)) d t}{\left[H^{\prime}(t)\right]^{2}} \\
& \left.+\frac{k}{i h^{2}} \int_{d}^{c} \frac{Q(t) G^{\prime \prime}(t) \exp (i h H(t)) d t}{\left[H^{\prime}(t)\right]^{2}}\right\} \\
= & 0\left(h^{\gamma-\lambda / \lambda}\right)+A+J^{\prime}(h, k)+J^{\prime \prime}(h, k)+J^{\prime \prime \prime}(h, k), \text { respectively. }
\end{aligned}
$$

Hence $|A| \leqq 2 M / m b^{\lambda-\gamma}=B b^{\gamma-\lambda}$,

$$
\begin{aligned}
\left|J^{\prime}(h, k)\right| & \leqq \frac{2 M h^{\gamma / \lambda}}{m h} \int_{d}^{c} t^{\gamma-\lambda-1} d t<\frac{2 M}{m(\lambda-\gamma) b^{\lambda-\gamma}}=B^{\prime} b^{\gamma-\lambda}, \\
\left|J^{\prime \prime}(h, k)\right| & \leqq \frac{4 M^{2}}{m^{2}(\lambda-\gamma) b^{\lambda-\gamma}}=B^{\prime \prime} b^{\gamma-\lambda}, \text { and } \\
\left|J^{\prime \prime \prime}(h, k)\right| & \leqq \frac{4 M^{2} k h^{-\gamma / \lambda}}{m^{2}(2 \lambda-\gamma-\nu) b^{2 \lambda-\gamma-\nu}} \leqq \frac{8 M^{2} \omega}{m^{2}(2 \lambda-\gamma-\nu) b^{\lambda-\gamma}}=B^{\prime \prime \prime} b^{\gamma-\lambda}
\end{aligned}
$$

Define

$$
h^{\gamma / \lambda} I_{0}(h, k)=\int_{0}^{\infty} x^{\gamma-1} q(0) \exp \left[i\left(f(0) x^{\lambda}+p g(0) x^{\nu}\right)\right] d x=\int_{0}^{b}+R(b) .
$$

Then there exists a number $K$ which is independent of $h, k$ and $\varepsilon$ and for which $|J(h, k)| \leqq K b^{\gamma-\lambda}$ and $|R(b)| \leqq K b^{\gamma-\lambda}$. Consider

$$
\begin{aligned}
(*) h^{\gamma / \lambda}\left(I_{0}-I\right)= & \int_{0}^{b} x^{\gamma-1}(q(0)-\widetilde{q}(x)) \exp \left[i\left(\widetilde{f}(x) x^{\lambda}+p \widetilde{g}(x) x^{\nu}\right)\right] d x \\
& +q(0) \int_{0}^{b} x^{\gamma-1}(1-P(x)) \exp \left[i\left(f(0) x^{\lambda}+p g(0) x^{\nu}\right)\right] d x \\
& +R(b)+0\left(h^{\gamma-\lambda / \lambda}\right)-J(h, k) \\
= & L(h, k)+L^{\prime}(h, k)+R(b)+0\left(h^{\gamma-\lambda / \lambda}\right)-J(h, k),
\end{aligned}
$$

respectively,

where $\left.P(x)=\exp \left\{i[\tilde{f}(x)-f(0)) x^{\lambda}+p(\widetilde{g}(x)-g(0)) x^{\nu}\right]\right\}$. By the choice 
of $c$ for each $\varepsilon>0$ we have $|L(h, k)| \leqq M \varepsilon b^{\lambda+\gamma},\left|L^{\prime}(h, k)\right|<2 M \varepsilon b^{\lambda+\gamma}+$ $2 M \varepsilon \omega b^{\nu+\gamma}$. If we take $\lim$ sup of both sides of $\left({ }^{*}\right)$ as $h, k \rightarrow \infty$ we obtain

$$
0 \leqq \lim \sup h^{\gamma / \lambda}\left|I_{0}-I\right| \leqq 3 M \varepsilon b^{\lambda+\gamma}+2 M \varepsilon b^{\nu+\gamma}+2 K b^{\gamma-\lambda}
$$

which is true for $\varepsilon>0$ and $b>N$. Since $\lim \sup h^{\gamma / \lambda}\left|I_{0}-I\right|$ is independent of both $\varepsilon$ and $b$ we first let $\varepsilon \rightarrow 0$ and then $b \rightarrow \infty$. Hence $h^{\gamma / \lambda}\left(I_{0}-I\right)=o(1)$ which implies that $I(h, k) \backsim I_{0}(h, k)$. To obtain the alternate form of $I_{0}(h, k)$ we let $x=h^{1 / \lambda}(k / h)^{1 /(\lambda-\nu)} t$.

5. Discussion of the suggested application. Consider for $x>0$ Schlafli's generalization of Bessel's integral:

$$
\begin{aligned}
J_{\nu}(x) & =\frac{1}{\pi} \int_{0}^{\pi} \cos (\nu t-x \sin t) d t-\frac{\sin \nu \pi}{\pi} \int_{0}^{\infty} \exp [-\nu t-x \sin h t] d t \\
& =\frac{1}{\pi} R \int_{0}^{\pi} \exp [i(\nu t-x \sin t)] d t+0\left(\frac{1}{\nu}\right) .
\end{aligned}
$$

Let $F^{\prime}(t)=t-\sin t$ and $|G(t)|=t$. We rewrite $F(t)$ as $F(t)=(1 / 6) t^{3}$ $\cos (r(t))$ and let $h=x, k=|\nu-x|, q(t) \equiv 1$ and $f(t)=1 \backslash 6 \cos (r(t))$. It follows that the condition $3 f(t)+t f^{\prime}(t)>0$ for $0 \leqq t \leqq \pi$ is satisfied since $F^{\prime}(t)=1-\cos t>0$ for $0<t \leqq \pi$.

We note that our Theorem 1 and III yield the dominant terms of some well known complete asymptotic expansions for $J_{\nu}(x)$ with $\tau=$ Arc$\cos \nu / x$ in Theorem III ${ }^{1}$. For the case $0<\lim \sup x^{-1 / 3}|\nu-x|<\infty$ we have by Theorem IV with $p=x^{-1 / 3}\left|(\nu-x) x^{-1 / 3}\right|$ that

$$
J_{\nu}(x) \backsim \frac{1}{\pi x^{1 / 3}} \int_{0}^{\infty} \cos \left(\frac{1}{6} t^{3}+p t\right) d t
$$

where the expression on the right is one of Airy's integrals ${ }^{2}$, whose evaluation for $p>0$ and $p<0$ yields precisely Nicholson's formulas when $\nu$ is an integer ${ }^{3}$.

${ }^{1}$ See W. Magnus and F. Oberhettinger, "Formeln und Satze fur die Speziellen Funktionen der Mathematischen Physik," Springer-Verlag, Berlin, 1948, pp. 33-34. Our theorems I and III give results which are equivalent to the dominant terms of the expansions $\left(b_{3}\right)$ and $\left(b_{1}\right)$, respectively.

"See, for example, G. N. Watson, "Theory of Bessel Functions," Cambridge, 1944, pp. $188-190$.

${ }^{3}$ See G. N. Watson, op. cit., pp. 248-249. 



\section{PACIFIC JOURNAL OF MATHEMATICS}

\section{EDITORS}

\author{
Ralph S. Phillips \\ Stanford University \\ Stanford, California \\ M. G. Arsove \\ University of Washington \\ Seattle 5 , Washington
}

\author{
A. L. Whiteman \\ University of Southern California \\ Los Angeles 7, California \\ Lowell J. Paige \\ University of California \\ Los Angeles 24, California
}
E. F. BECKENBACH
D. DERRY
ASSOCIATE EDITORS
T. M. CHERRY
M. OHTSUKA
H. L. ROYDEN
E. G. STRAUS
E. SPANIER
F. WOLF

\section{SUPPORTING INSTITUTIONS}

\author{
UNIVERSITY OF BRITISH COLUMBIA \\ CALIFORNIA INSTITUTE OF TECHNOLOGY \\ UNIVERSITY OF CALIFORNIA \\ MONTANA STATE UNIVERSITY \\ UNIVERSITY OF NEVADA \\ NEW MEXICO STATE UNIVERSITY \\ OREGON STATE UNIVERSITY \\ UNIVERSITY OF OREGON \\ OSAKA UNIVERSITY \\ UNIVERSITY OF SOUTHERN CALIFORNIA
}

\author{
STANFORD UNIVERSITY \\ UNIVERSITY OF TOKYO \\ UNIVERSITY OF UTAH \\ WASHINGTON STATE UNIVERSITY \\ UNIVERSITY OF WASHINGTON \\ AMERICAN MATHEMATICAL SOCIETY \\ CALIFORNIA RESEARCH CORPORATION \\ SPACE TECHNOLOGY LABORATORIES \\ NAVAL ORDNANCE TEST STATION
}

Mathematical papers intended for publication in the Pacific Journal of Mathematıcs should be typewritten (double spaced), and the author should keep a complete copy. Manuscripts may be sent to any one of the four editors. All other communications to the editors should be addressed to the managing editor, L. J. Paige at the University of California, Los Angeles 24, California.

50 reprints per author of each article are furnished free of charge; additional copies may be obtained at cost in multiples of 50 .

The Pacific Journal of Mathematics is published quarterly, in March, June, September, and December. Effective with Volume 13 the price per volume (4 numbers) is $\$ 18.00$; single issues, $\$ 5.00$. Special price for current issues to individual faculty members of supporting institutions and to individual members of the American Mathematical Society: $\$ 8.00$ per volume; single issues $\$ 2.50$. Back numbers are available.

Subscriptions, orders for back numbers, and changes of address should be sent to Pacific Journal of Mathematics, 103 Highland Boulevard, Berkeley 8, California.

Printed at Kokusai Bunken Insatsusha (International Academic Printing Co., Ltd.), No. 6, 2-chome, Fujimi-cho, Chiyoda-ku, Tokyo, Japan.

PUBLISHED BY PACIFIC JOURNAL OF MATHEMATICS, A NON-PROFIT CORPORATION

The Supporting Institutions listed above contribute to the cost of publication of this Journal, but they are not owners or publishers and have no responsibility for its content or policies. 


\section{Pacific Journal of Mathematics}

\section{Vol. 12 , No. 4 \\ April, 1962}

Tsuyoshi Andô, On fundamental properties of a Banach space with a cone ..... . 1163

Sterling K. Berberian, A note on hyponormal operators ................ 1171

Errett Albert Bishop, Analytic functions with values in a Frechet space . . . . . . . 1177

(Sherman) Elwood Bohn, Equicontinuity of solutions of a quasi-linear equation ............................................ 1193

Andrew Michael Bruckner and E. Ostrow, Some function classes related to the class of convex functions . . . . . . . . . . . . . . . . . . . . . . . . 1203

J. H. Curtiss, Limits and bounds for divided differences on a Jordan curve in the complex domain . . ................................. 1217

P. H. Doyle, III and John Gilbert Hocking, Dimensional invertibility . . . . . . . . 1235

David G. Feingold and Richard Steven Varga, Block diagonally dominant matrices and generalizations of the Gerschgorin circle theorem ................. 1241

Leonard Dubois Fountain and Lloyd Kenneth Jackson, A generalized solution of the boundary value problem for $y^{\prime \prime}=f\left(x, y, y^{\prime}\right) \ldots \ldots \ldots \ldots \ldots \ldots \ldots \ldots \ldots$

Robert William Gilmer, Jr., Rings in which semi-primary ideals are primary. . . . . 1273

Ruth Goodman, K-polar polynomials .......................... 1277

Israel Halperin and Maria Wonenburger, On the additivity of lattice completeness ........................................... 1289

Robert Winship Heath, Arc-wise connectedness in semi-metric spaces . . . . . . . . 1301

Isidore Heller and Alan Jerome Hoffman, On unimodular matrices . . . . . . . . . . . 1321

Robert G. Heyneman, Duality in general ergodic theory . . . . . . . . . . . . . . . 1329

Charles Ray Hobby, Abelian subgroups of p-groups . . . . . . . . . . . . . . . 1343

Kenneth Myron Hoffman and Hugo Rossi, The minimum boundary for an analytic

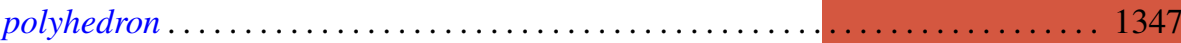

Adam Koranyi, The Bergman kernel function for tubes over convex cones ........ 1355

Pesi Rustom Masani and Jack Max Robertson, The time-domain analysis of a continuous parameter weakly stationary stochastic proces.

William Schumacher Massey, Non-existence of almost-complex structures on

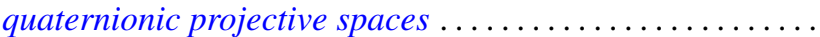

Deane Montgomery and Chung-Tao Yang, A theorem on the action of $\mathrm{SO}(3) \ldots . .1385$

Ronald John Nunke, A note on Abelian group extensions . . . . . . . . . . . . . 1401

Carl Mark Pearcy, A complete set of unitary invariants for operators generating finite $W^{*}$-algebras of type $I$

Edward C. Posner, Integral closure of rings of solutions of linear differential equations.

Duane Sather, Asymptotics. III. Stationary phase for two parameters with an application to Bessel functions.

J. Śladkowska, Bounds of analytic functions of two complex variables in domains

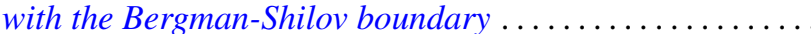

Joseph Gail Stampfli, Hyponormal operators .

George Gustave Weill, Some extremal properties of linear combinations of kernels

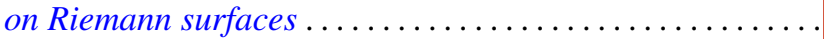

